\title{
émulations
}

\section{Yasmine Bouagga - De Lesbos à Calais : comment l'Europe fabrique des camps}

Alise Dauchy

Émulations - Revue de sciences sociales

2019. Comptes rendus critiques. En ligne

\section{Article disponible à l'adresse suivante}

https://ojs.uclouvain.be/index.php/emulations/article/view/7483

\section{Pour citer cet article}

Alise Dauchy, « Yasmine Bouagga - De Lesbos à Calais : comment l'Europe fabrique des camps », Émulations, en ligne. Mise en ligne le 10 février 2019.

DOI : 10.14428/emulations.cr.047

Distribution électronique : Université catholique de Louvain (Belgique) : ojs.uclouvain.be

(C) Cet article est mis à disposition selon les termes de la Licence Creative Commons Attribution, Pas d'Utilisation Commerciale 4.0 International. http://creativecommons.org/licenses/by$\mathrm{nc} / 4.0 /$

Éditeur : Émulations - Revue de sciences sociales / Presses universitaires de Louvain https://ojs.uclouvain.be/index.php/emulations

ISSN électronique : 1784-5734

UCL PRESSES

UNIVERSITAIRES 


\title{
Yasmine Bouagga (dir.) - De Lesbos à Ca- lais : comment l'Europe fabrique des

\begin{abstract}
Alise Dauchy ${ }^{1}$
Recensé : Yasmine Bouagga (dir.), De Lesbos à Calais. Comment l'Europe fabrique des camps, Neuvy-en-Champagne, Le Passager clandestin, coll. «Babels», 2017, 152 p.

De Lesbos à Calais : Comment l'Europe fabrique des camps est un des sept titres de la collection "Bibliothèque des frontières", qui rassemble des publications de chercheur.e's réuni.e's autour de Michel Agier, dans le cadre du programme de recherche Babels ${ }^{2}$. Cette collection invite le lecteur à s'interroger sur les modalités de gestion des migrations internationales contemporaines. Yasmine Bouagga coordonne ici une étude sur les camps en Europe à partir de terrains menés par quinze auteur.e-s qui nous donnent accès à leurs carnets de recherches. On ne s'étonne pas du choix de la maison d'édition indépendante "Le passager clandestin", tant les chercheur.e.s nous offrent, en sus de leurs analyses, une photographie des luttes et des mobilisations solidaires des étranger·es en cours sur le territoire européen.

L'ouvrage est divisé en cinq parties et chacune d'entre-elles rassemble trois sous-parties, telles des étapes sur un itinéraire partant de Lesbos et allant jusqu'à VintimilleMenton, passant par Lampedusa et Paris, pour arriver à Calais. Dès l'introduction, les récits de ces « lieux-frontières » ou de ces «villes-refuges » s'entremêlent, marquant les similitudes et les différences des situations d'encampement. Notons que les auteur.e-s font le choix de ne pas signer l'écriture des différents chapitres.

Les enjeux soulevés dans ces études dépassent une analyse au cas par cas suivant le type de camp : Hotspot, centre de premier secours et d'accueil, centre de rétention administrative (CRA), centre d'accueil et d'orientation, camp humanitaire - officiel ou campement de rue sauvage -, squat et bidonville, etc. Il serait abusif de faire fi des différents statuts administratifs accolés à ces dispositifs, néanmoins il apparait au fil de la lecture que les pratiques des acteurs reflètent mieux la nature de ces camps qu'une tentative de classification. Les auteur.e-s s'appliquent ainsi à présenter la pluralité des acteurs : les migrant.e.s et leur soutien, les riverains de ces camps, les corps de police
\end{abstract}

\footnotetext{
${ }^{1}$ Chercheure à l'Université Saint-Louis à Bruxelles.

2 Michel Agier et Babels - Le passager clandestin: http://lepassagerclandestin.fr/auteurs/a/michel-agier-et-babels.html.
} 
variés, les associations, les organisations non gouvernementales et internationales·e·s, les sociétés privés...

En cela, une lecture approfondie de l'ouvrage peut mener à dégager plusieurs enseignements que nous proposons de regrouper au sein de deux parties. Premièrement, «les camps » sont le symptôme d'un manque d'anticipation qui structure les politiques migratoires menées par les États européens. Deuxièmement, ils constituent des lieux où s'exerce par intermittence un "pouvoir protecteur » et un "pouvoir répressif» de la part d'acteurs dont les intérêts peuvent converger.

\section{Le camp : une infrastructure ${ }^{3}$ européenne}

Les auteur.e-s font état des multiples objectifs accolés aux camps mis en place par les États membres depuis les années 1990. Pour saisir leurs conditions de "fabrication", les sous-parties sont étoffées et accompagnées d'encadrés qui explorent des questions spécifiques. L'objectif est d'éclairer le lecteur sur les conditions de mise en œuvre des politiques migratoires, en expliquant les contextes sibyllins où s'enchevêtrent des enjeux locaux, entre les États membres et internationaux. Ainsi, la première partie intitulée «Lesbos. L'identification et le tri aux frontières de l'Europe» comprend une présentation des premières politiques migratoires espagnoles mises en place dès les années 1980. Ce détour par l'Espagne apporte des éléments de compréhension des politiques d'accueil et de contrôle face au phénomène des boat people débarquant sur les rives européennes, mais aussi des éléments plus factuels, comme le rôle de la météo. À titre d'exemple, celle-ci y est décrite comme un facteur d'affluence des migrant-e·s; les tentatives de traversées sont en effet moins fréquentes en hiver que lorsque la mer est plus calme, à la belle saison. Un constat trivial qui permet pourtant au lecteur de se familiariser avec les pratiques migratoires.

L'ouvrage adopte une approche ethnographique, d'une part car les auteure-s s'inscrivent dans le temps long sur leurs terrains et qu'ils et elles participent activement aux mobilisations en cours, d'autre part en raison du matériel proposé dans le livre : cartes, photographies, témoignages et illustrations. Cependant, l'ouvrage ne renonce pas à inscrire le camp dans une dimension internationale, à montrer comment le territoire local est tributaire d'une gouvernance globale des migrations. Tel est à cet égard l'un des enseignements de la deuxième partie intitulée «La frontière Vintimille-Menton. Derrière la "crise", la continuité de la forme-camp et des contrôles frontaliers ». On y apprend que les policiers français interdisent aux migrant·es de traverser la frontière, notamment, car «la situation calaisienne dicte les orientations prises à la frontière sud» (p. 56). À Vintimille, l’objectif des autorités françaises serait donc moins de blo-

\footnotetext{
${ }^{3}$ Larkin, 2013.
} 
quer les personnes sur le long terme, que de ralentir leur arrivée dans les régions parisienne et calaisienne. L'encampement est présenté par les auteur.e-s - quels que soient les terrains d'enquête - comme une réponse ponctuelle, située et technique. La grande sensibilité dont ils font part dans leurs récits des vies des personnes exilées aux frontières européennes met d'ailleurs l'accent sur les conséquences de ces solutions précaires sur la condition des migrant.e.s. Ils et elles relèvent la place importante accordée à l'expérience de l'enfermement dans le discours et le vécu du migrant. Le lecteur assiste ainsi à un aller-retour continu et rythmé entre le camp et les enjeux internationaux dans lesquels il s'inscrit.

De plus, on pourrait être surpris de la duplicité des objectifs poursuivis par les camps en Grèce et en Italie. Dans ces deux pays d'arrivées des migrant-ess par la mer, on apprend que, si les camps servent à mettre à l'abri provisoirement, ils sont également utilisés par les différents acteurs (autorités locales, nationales, force de l'ordre, agence européenne Frontex) pour identifier les personnes en migration, à partir d'entretien et de procédure biométrique telle que la prise d'empreinte digitale. « De Vincennes à Lampedusa », pour reprendre en partie le nom de la troisième partie, nous rappelle que cette récolte d'informations ne vise pas seulement à identifier les personnes les plus vulnérables, mais à opérer un tri entre demandeurs d'asile et «migrants économiques », avec l'objectif latent d'éloigner les seconds.

Grâce à une large connaissance de leurs terrains, les auteur.e's percent d'autres fonctions des camps, et partagent ainsi avec le lecteur une analyse que l'on peut résumer en ces termes : le camp n'est pas seulement un outil technique visant à accueillir ou à interdire des personnes sur un territoire, c'est un lieu où le pouvoir des gouvernants s'exerce par le biais de procédures d'identification et d'enregistrement.

Les contrôles effectués par la police aux frontières situées sur l'axe Menton-Vintimille évoquent également un autre type de relation entre le politique et le camp, audelà de la fonction traditionnelle de «blocage». La mission des forces de l'ordre à la frontière consiste à garantir la liberté de circulation des citoyens européens au sein de l'espace Schengen, et pour cela, ils associent «un récit imaginaire stéréotypé» (p. 48) aux personnes souhaitant traverser la frontière. Ils s'appuient sur des caractéristiques qui seraient propres aux migrant-e-s pour les empêcher de circuler. Certes les campements se forment à mesure que les migrant-e-s décident de s'installer à la frontière. Mais ce truisme ne doit pas mener à considérer l'installation de campements comme des événements inopinés et fortuits. Les auteur.e-s nous invitent à considérer le renforcement des contrôles aux frontières comme un acte opéré de manière discrétionnaire, mais aussi comme un projet qui préexiste à l'installation des camps, ici, celui de réguler, ralentir voire suspendre le projet de mobilité des migrant.e.s.

En outre, en capitalisant des connaissances et en acquérant un savoir-faire, le camp participe à l'ajustement et à la définition des politiques publiques dans un monde globalisé. Les auteur.e-s amènent le lecteur à considérer le camp au-delà de sa simple fonction «d'accueil» sur la route des migrant·e-s. Le camp est un espace de médiation qui 
traduit une inquiétude et le besoin pour les autorités de réguler la circulation par l'emploi d'une infrastructure (Larkin, 2013) ; une construction matérielle et humaine qui façonne notamment la vitesse et la direction des flux. Il ne doit donc pas seulement être envisagé comme un dispositif d'urgence répondant à une situation exceptionnelle en raison d'un manque d'anticipation des autorités. En s'inspirant des recherches en politiques publiques, on peut ajouter que le camp appartient également à un ensemble plus large de mesures visant à contrôler, et définies au fil des années par les pouvoirs publics (Boussaguet, Jacquot, Ravinet, 2014).

\section{Mise en œuvre d'un « pouvoir protecteur » et d'un « pouvoir répressif » au sein du camp}

Les auteur.e-s interrogent la nature des camps, et les différentes modalités de fonctionnement. Au cœur de l'ouvrage, la troisième partie sur le «business » de l'enfermement des étrangers propose un retour sur les origines de la rétention administrative en France. On y apprend que la rétention des individus a d'abord été définie par la loi Questiaux en 1981; celle-ci s'adressait aux individus subversifs avant leur expulsion du territoire français, notamment dans le contexte de la guerre d'Algérie. Pour ce qui est de l'enfermement de ressortissants d'États tiers en situation administrative irrégulière, «le droit est ainsi venu a posteriori, encadrer des pratiques déjà en cours» (p. 64). En outre, il est précisé que la durée de détention avant l'expulsion - qui n’a cessé d'être rallongée - n'a pas été davantage suivie d'expulsions massives et systématiques. Cette approche historique présente plusieurs mérites. D'abord, elle questionne la nature de la détention administrative : il ne s'agit pas d'une pratique neutre, elle est d'ailleurs née dans le contexte particulier de la décolonisation et des luttes pour l'indépendance. Puis, la détention aussi semble davantage avoir pour effet de ralentir la mobilité des personnes en les interceptant et en les enfermant, que de diminuer le nombre d'étranger-e·s sur le territoire; en témoigne l'écart entre les objectifs déclarés et les objectifs atteints de la rétention (p. 65). Enfin, la troisième partie s'intéresse également au développement d'un marché du contrôle et de la sécurité attirant des intérêts privés. Cette incision sur la question d'un marché de l'enfermement (Rodier, 2012), apporte au lecteur les tenants et les aboutissants d'une question encore subsidiaire dans la littérature francophone (Gammeltoft-Hansen, Nyberg Sorensen, 2013).

Si la gestion du CRA de Vincennes diffère dans ses modalités opératoires des camps gérés par la Croix-Rouge à Vintimille, ou par des coopératives à Lampedusa, le collectif Babels met en exergue une gestion à la fois humanitaire et répressive des camps. Qu'ils soient " ouverts » ou «fermés », ces lieux sont marqués par des contraintes qui pèsent à la fois et principalement sur les exilée-es, mais aussi sur les opérateurs des associations ou des entreprises en charge de leur fonctionnement quotidien. Le lecteur constatera par ailleurs que les camps semblent avoir en commun une organisation qui s'appuie sur un traitement différentiel des migrant·e-s, selon qu'ils sont demandeurs d'asile ou 
«migrant économique». Cette catégorisation parait être étroitement liée aux différentes procédures juridiques auxquelles sont soumis les migrant·e-s, et qui sont mises en œuvre par des ONG et les agences onusiennes (Organisation Internationale pour les Migrations - OIM, Haut-Commissariat des Nations unies pour les Réfugiés - UNHCR) (Pécoud, 2017). En cela, une étude approfondie des profils et des parcours des acteurs - leurs appartenances à différents contextes socio-économique selon qu'ils sont des opérateurs locaux ou internationaux - offrirait un regard nouveau sur les pratiques quotidiennes dans le camp et les enjeux qu'il recouvre. Cette démarche est initiée à la fin de l'ouvrage, on note à ce titre un encadré intitulé "Secourir hors des camps : le questionnement d'un acteur humanitaire», dans la cinquième partie «Paris : campements de rue, mobilisations citoyennes et politiques de la dispersion ». Cet entretien avec un responsable de MSF répond en partie au besoin que le lecteur pourrait ressentir d'une démonstration entière et complète de l'impact des trajectoires individuelles des acteurs dans leur manière d'appréhender les camps et d'assoir la légitimité et la pérennisation de ces lieux. Pour autant, l'ouvrage fait la part belle aux exemples de solidarités et de contestation ; la mobilisation se politise en effet à mesure que les « solutions d'urgence routinisées » (p.114) se révèlent inefficaces. On trouve des exemples de communautés de vie qui s'organisent selon une approche horizontale visant l'autogestion et la prise de décision collective. Pour autant, les exposés ne masquent pas les clivages et les tensions avec le voisinage notamment, ainsi que la violence propre à ces lieux.

Enfin, les auteur.e-s s'intéressent à l'acteur étatique dans ses diverses manifestations dans les quatrième et cinquième parties intitulées respectivement, «Calais. Campements et camps calaisiens de l'enfermement des étrangers », et « Paris. Campements de rue, mobilisations citoyennes et politiques de la dispersion ». Les autorités adopteraient tantôt des mesures sécuritaires visant à rassurer le citoyen de la menace que représenterait une invasion de migrants. Ces opérations sont qualifiées de " mises à l'abri » (p. 83 et 120) et se traduisent par l'évacuation de campements en vue de réaménager l'espace public pour empêcher toute réinstallation. Ainsi, en lieu et place d'une réflexion sur le long terme et d'un engagement de manière à « fixer ces populations dans un nouvel espace où elles pourraient trouver leur place» (p. 57), l'ouvrage témoigne de l'attitude fluctuante des autorités qui tentent d'effacer les lieux de regroupement des migrant·e·s, sous couvert de discours à la fois bienveillants et sécuritaires.

Au-delà du caractère minutieux de leurs analyses, les auteur.e.s partagent une même sensibilité dans leur manière de rendre compte du vécu des exilée.e-s dans l'intimité des camps. Ils et elles s'attachent à relayer leurs combats mais aussi à nous faire adopter le point de vue des personnes en migration, et en particulier d'un sentiment diffus parmi elles, celui de se sentir moins dépossédée de son libre-arbitre au sein des campements informels que dans des lieux de détention où les sentiments d'ennui et de résignation 
sont plus forts. Ainsi, l'on constate que ce sont moins les conditions de vie que les modalités d'occupation de l'espace et d'organisation qui différencient les camps entre eux.

De nombreuses questions sont soulevées au fil des chapitres, nous avons choisi d'en problématiser certaines afin de montrer toute la pertinence et l'actualité de cet ouvrage. Néanmoins, plusieurs lectures peuvent être faites en fonction des connaissances préalables du lecteur sur la question des camps. On apprécie à cet égard le caractère accessible de l'ouvrage à un large public, grâce notamment à des encadrés explicatifs et à la présence d'illustrations qui rendent la lecture vivante et au plus près du terrain. Il faut noter que le format de l'ouvrage n'est cependant pas toujours adapté à certaines illustrations qui sont malheureusement peu lisibles.

Néanmoins, cette accessibilité tend parfois à noyer les arguments des auteur.e-s dans une suite de récits laissant le lecteur averti sur sa fin. L'on regrette de ne pas pouvoir identifier de manière claire les auteur.e.s à l'initiative de chacune des parties, mais aussi de ne pas assister à un dialogue plus sagace entre eux sur les questions transversales qu'ils et elles amènent à partir de leurs expériences dans les différents pays membres. Ainsi, la question des politiques « d'accueil» des étranger-e.s apparaît en filigrane, pour autant les auteur.e.s manquent une occasion d'échanger et de réfléchir ensemble à l'état et au devenir de l'hospitalité en Europe à partir des diverses manifestations d'hostilité qu'ils décrivent scrupuleusement. La brièveté de l'ouvrage ne se prête peut-être pas à ce double exercice. Cette hypothèse pourrait être affinée à la lecture des autres titres de la collection, notamment celui publié en janvier 2018, «Entre accueil et rejet : ce que les villes font aux migrants » (Bontemps, Makaremi, Mazouz, 2018).

\section{Bibliographie}

AgieR M. (2002), Aux bords du monde, les réfugiés, Paris Flammarion.

Bontemps V., MAKAREMi C., Mazouz S. (dir.) (2018), Entre accueil et rejet: ce que les villes font aux migrants, Lyon, Le Passager Clandestin.

Bouagga Y. (dir.) (2017), De Lesbos à Calais. Comment l'Europe fabrique des camps, Neuvy-en-Champagne, Le Passager Clandestin.

Boussaguet L., JaCquot S., RAvinet P. (2014), Dictionnaire des politiques publiques, $4^{\text {ème }}$ édition, Presses de Sciences Po.

FAssin D. (2010), La raison humanitaire, une histoire morale du temps présent, Paris, Gallimard Seuil.

Gammeltoft-Hansen T., Nyberg Sorensen N. (2013), The Migration Industry and theCommercialization of International Migration, Routledge.

LARKin B. (2013), "The Politics and Poetics of Infrastructure », Annual Review of Anthropology, ${ }^{\circ}$ 42, p. 327-343. 
PARIzOT C. (2018), «Viscous Spatialities: The Spaces of the Israeli Permit Regime of Access and Movement », The South Atlantic Quarterly, n¹17, p. 21-42.

PeCoud A. (2017), « De la "gestion” au contrôle des migrations ? Discours et pratiques de l'Organisation Internationale pour les Migrations», Critique internationale, vol. 76, $\mathrm{n}^{\circ} 3$, p. 81-99.

RoDier C. (2012), Xénophobie Business, Paris, La Découverte. 